Hautarzt 2020 $71: 405-406$

https://doi.org/10.1007/s00105-020-04595-2

(C) Springer Medizin Verlag GmbH, ein Teil von Springer Nature 2020

\section{P. Elsner ${ }^{1} \cdot$ M. Fischer ${ }^{2} \cdot$ G. Weisel ${ }^{3}$}

'Klinik für Hautkrankheiten, Universitätsklinikum Jena, Jena, Deutschland

${ }^{2}$ Klinik für Dermatologie, Venerologie und Allergologie, Bundeswehrkrankenhaus Hamburg, Hamburg, Deutschland

${ }^{3}$ Dermatologie, Venerologie und Allergologie, Bundeswehrkrankenhaus Ulm, Ulm, Deutschland

\title{
Dermatologie im Sanitätsdienst der Bundeswehr - ein essenzielles Fachgebiet
} tionen und Beatmungskapazitäten überfordern könnten. Es sind Szenarien wie in Kriegszeiten, die nur noch ältere Menschen in Deutschland aus eigenem Erleben kennen.

Inzwischen wurde die Katastrophenlage von einzelnen Bundesländern und Gemeinden ausgerufen, und wie bei Katastrophen in der Vergangenheit ertönt der Ruf nach medizinischer und logistischer Unterstützung durch die Bundeswehr.

Die Aufgabe der deutschen Streitkräfte, die in langen Friedenszeiten von manchem als verzichtbar eingeschätzt wurden und an deren zeitgemäßer Ausstattung lange gespart wurde, gerät plötzlich wieder in den Blick: Sie haben unser Land nicht nur nach außen im Rahmen kollektiver Bündnisse zu verteidigen und in internationalen Missionen friedenserhaltend tätig zu sein, sondern auch die Aufgabe, nach Art. 35 Grundgesetz bei Katastrophen und schweren Unglücksfällen Behörden des Bundes und der Länder Amtshilfe zu leisten. Diese Aufgabe zur Daseinsvorsorge in unserem Gemeinwesen teilt die Bundeswehr mit dem zivilen Gesundheitswesen, das auch mehr ist als ein oftmals gescholtener Kostenfaktor für die Volkswirtschaft, wie die aktuelle Pandemie schmerzhaft verdeutlicht.

\section{\) Die Dermatologie ist aufgrund ihrer Bedeutung für die Einsatzfähigkeit der Bundeswehr als Fachgebiet im Sanitätsdienst vertreten}

breitete infektiöse Hautkrankheiten und sexuell übertragene Infektionen.

Gerade bei den internationalen Einsätzen der Bundeswehr in den letzten Jahrzehnten wurden und werden Fachärzte für Haut- und Geschlechtskrankheiten gebraucht für

- die fachlich dermatologische Beratung der Sanitätsführung in der Planung von Einsätzen unter präventiven Gesichtspunkten,

- die Beurteilung der Tauglichkeit von Soldaten für spezifische Einsatz-

Menschen, die in der Bundeswehr Dienst tun, haben Anspruch auf eine medizinische Versorgung, die im Ergebnis der im zivilen Gesundheitswesen entspricht. Dazu hält die Bundeswehr einen Sanitätsdienst bereit, der aufgrund seiner Ausstattung und Qualität seiner Soldaten internationales Ansehen genießt. Bis auf die Geburtsheilkunde und Gynäkologie (mit Ausnahme einer fachärztlichen Untersuchungsstelle am Bundeswehrkrankenhaus Westerstede) und der Pädiatrie sind im Sanitätsdienst alle medizinischen Fachgebiete vertreten. Die Dermatologie, obgleich primär eine nichttraumatologische Disziplin, gehört aufgrund ihrer besonderen Bedeutung für die Einsatzfähigkeit von Soldaten seit ihrer Etablierung als Fachgebiet dazu. Während in den Weltkriegen des vergangenen Jahrhunderts insbesondere die sexuell übertragbaren Erkrankungen die Militärdermatologen forderten, sind es im Jahr 2020 neben den auch im Inland häufigen Dermatosen in tropischen Einsatzregionen ver- regionen unter dermatologischen Aspekten,

- die einsatzbezogene Individualprävention bei Soldaten mit vorbestehenden Hautkrankheiten (z. B. Akne, atopisches Ekzem, Allergien) und

- die Erhaltung und Wiederherstellung der Dienstfähigkeit im Einsatz beim akutem Auftreten von Haut- und Geschlechtskrankheiten im Einsatzgebiet oder bei Verschlechterung vorbestehender Dermatosen sowie

- die Mitwirkung im Bereich der Behandlung komplizierter Wunden einschließlich Verbrennungen.

Bis zu 25\% aller Konsultationen durch militärisches Personal in tropischen Einsatzgebieten betreffen die Dermatologie [1].

Die erforderlichen einsatzbezogenen Kenntnisse und Fertigkeiten für den Facharzt für Haut- und Geschlechtskrankheiten werden in der zivilen Weiterbildung zum Dermatologen in Deutschland nur unzureichend entwi- 
ckelt. Selbst Kliniken, die den von der Deutschen Dermatologischen Gesellschaft geforderten Mindeststandards für dermatologische Kliniken entsprechen, verfügen vielfach nicht über die nötige Kompetenz, geschweige denn das für die Weiterbildung erforderliche Patientenspektrum auf den Gebieten der dermatologischen Infektiologie, der Tropendermatologie und der Berufsdermatologie. Die Veränderung des Leistungsspektrums ziviler dermatologischen Kliniken unter DRG(Diagnosis Related Groups)-Aspekten und dermatologischer Weiterbildungspraxen unter dem Gesichtspunkt von kosmetischen Leistungen führt dazu, dass die Weiterbildung von für Einsätze der Bundeswehr qualifizierten Dermatologen im zivilen Bereich nicht gewährleistet ist.

\section{》) Dermatologen der Bundeswehr haben eine hohe Expertise besonders in der Tropen- und Reisedermatologie}

Die Bundeswehr verfügt mit ihren Abteilungen für Dermatologie in den Bundeswehrkrankenhäusern Berlin, Hamburg, Koblenz und Ulm daher über eigene Weiterbildungseinrichtungen, die nicht nur eine vollständige dermatologische Weiterbildung entsprechend den Weiterbildungsordnungen der Landesärztekammern ermöglichen, sondern auch die für einen Sanitätsoffizier im Einsatz zusätzlich erforderlichen Fähigkeiten vermitteln. Durch ihre internationalen Einsätze und die Kooperation mit dem Bernhard-Nocht-Institut für Tropenmedizin in Hamburg haben Dermatologen der Bundeswehr in den vergangenen Jahren eine einzigartige Expertise auf dem Gebiet der Tropen- und Reisedermatologie entwickelt. Konsequenterweise waren Bundeswehrdermatologen an der Etablierung der International Society of Dermatology in the Tropics (ISDT) und an der Einführung des Zertifikats für Tropen- und Reisedermatologie der Deutschen Dermatologischen Akademie (DDA) [2] führend beteiligt.

Von den besonderen Erfahrungen der dermatologischen Kolleginnen und Kol- legen in der Bundeswehr profitieren so nicht nur die deutschen Streitkräfte und die verbündeter Nationen, sondern unsere deutschsprachige Dermatologie als Ganzes.

Dieses Heft der Zeitschrift Der Hautarzt soll einen Überblick bieten über das Tätigkeitsspektrum der Dermatologie an den Hautkliniken der Bundeswehr, das gerade in Zeiten internationaler Migration für uns alle von Bedeutung ist.

Vielleicht regt es auch zivile Dermatologinnen und Dermatologen an, als Reservisten tätig zu werden. Die Sicherstellung des ärztlichen und so auch des dermatologischen Nachwuchses in der Bundeswehr wird seit Aufhebung der Wehrpflicht immer schwieriger. QuereinsteigerInnen in die Bundeswehrdermatologie sind daher gesucht und willkommen!

Prof. Dr. P. Elsner

Dr. M. Fischer

Dr. G. Weisel

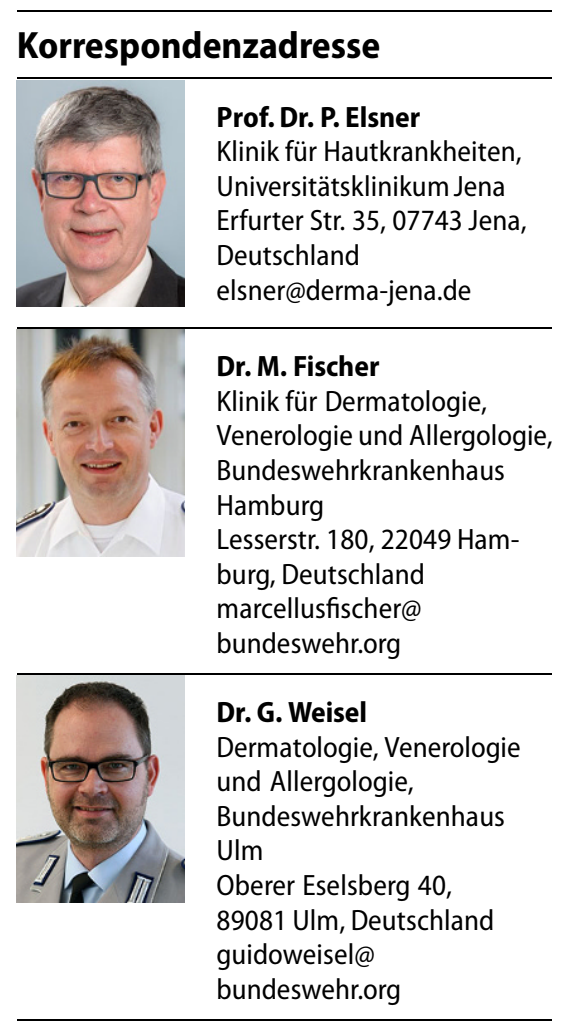

Interessenkonflikt. P. Elsner ist Mitglied des Wehrmedizinischen Beirats beim Bundesverteidigungsministerium. P. Elsner, M. Fischer und G. Weisel geben an, dass kein Interessenkonflikt besteht.

\section{Literatur}

1. Elsner P, Glitsch M, Merk HF (2015) Dermatology on international missions: Globalization of a medical specialty. Hautarzt 66:308-310

2. Elsner P, Nenoff P, Schliemann S et al (2013) Zertifizierung: „Tropen- und Reisedermatologie (DDA)": Seminar-Curriculum der Society for Dermatology in the Tropics in der ADI-TD. Aktuelle Derm 39:176-180 